\title{
CHARACTERIZATION OF $p$-PREDICTORS
}

\author{
D. LANDERS AND L. ROGGE
}

\begin{abstract}
Let $(\Omega, Q, P)$ be a probability space and $1<p<\infty$. It is shown that each operator $T: L_{p}(\Omega, \mathcal{Q}, P) \rightarrow L_{p}(\Omega, \mathcal{Q}, P)$ which is homogeneous, constant preserving, positive, quasi-additive and fulfills Dykstra's condition is an $p$-predictor with respect to a suitable $\sigma$-field, i.e. a nearest point projection onto a closed subspace $L_{p}(\Omega, \mathscr{B}, P)$, where $\mathscr{B} \subset \mathbb{Q}$ is a $\sigma$-field. None of the conditions for $T$ can be dispensed without compensation.
\end{abstract}

Let $(\Omega, Q, P)$ be a probability space and $L_{p}(\Omega, \mathcal{Q}, P)$ (for $1<p<\infty$ ) be the space of all $P$-equivalence classes of real-valued $Q$-measurable functions whose absolute $p$ th powers are integrable. Let $\mathscr{B}$ be a sub- $\sigma$-field of $\mathcal{Q}$, then $L_{p}(\Omega, \Re, P)$-or $L_{p}(\Im)$ for short-is the system of all equivalence classes of $L_{p}(\Omega, Q, P)$, containing a $\mathscr{B}$-measurable function. The operator $P_{p}^{\mathscr{B}_{B}}$ which assigns to each $f \in L_{p}(\Omega, Q, P)$ the unique element in $L_{p}(\Omega, \mathscr{B}, P)$ with minimum distance from $f$ is called the $p$-predictor given $\mathscr{B}$ (see Ando and Amemiya [2]). $P_{p}^{\mathscr{B}} f$ is the unique element of $L_{p}(\mathscr{B})$ with

$$
\left\|f-P_{p}^{\mathscr{B}} f\right\|_{p} \leqslant\|f-g\|_{p}
$$

for all $g \in L_{p}(\Re)$.

The operator $T=P_{p}^{\mathscr{B}}: L_{p}(\Omega, \mathcal{Q}, P) \rightarrow L_{p}(\Omega, \mathcal{Q}, P)$ has the following properties (see [2]):

(1) $T$ is homogeneous, i.e., $T(\alpha f)=\alpha T f$, for $f \in L_{p}(\Omega, Q, P)$ and $\alpha \in \mathbf{R}$;

(2) $T$ is quasi-additive, i.e., $T(f+T g)=T f+T g$, for $f, g \in L_{p}(\Omega, Q, P)$;

(3) $T$ is constant-preserving, i.e., $T 1=1$;

(4) $T$ is positive, i.e., $T f \geqslant 0$, for $0 \leqslant f \in L_{p}(\Omega, \mathbb{Q}, P)$;

(5) $T$ fulfills Dykstra's condition, i.e. (see [5]), $\|I-T\|_{p}<1$, where $\| I-$ $T \|_{p}$ is defined by

$$
\|I-T\|_{p}=\inf \left\{c:\|f-T f\|_{p}<c\|f\|_{p}\right\} .
$$

In the special case $p=2, P_{2}^{9} f$ is the usual conditional expectation of $f$ given $\mathscr{B}$ with respect to $P$. The operators $P_{2}^{\mathscr{B}}$ have been characterized by many authors, for instance Bahadur [3], Douglas [4], Moy [7] and Pfanzagl [9]. But as far as we know there does not exist a characterization for $P_{p}^{\mathscr{B}}$ if $p \neq 2$. This may be due to the fact that $P_{p}^{\mathscr{B}}$ is in general not a linear operator (for a characterization of linearity of $P_{p}^{\mathscr{B}}$ see [6]). Now we prove that every operator $T: L_{p}(\Omega, \mathcal{Q}, P) \rightarrow L_{p}(\Omega, \mathcal{Q}, P)$ which fulfills (1)-(5) is a $p$-predictor $P_{p}^{\mathscr{B}}$ for some suitable sub- $\sigma$-field $\mathscr{B} \subset \mathbb{Q}$.

Received by the editors September 14, 1978.

AMS (MOS) subject classifications (1970). Primary 60A05; Secondary 46E30, 47B99.

$K e y$ words and phrases. Projection, $L_{p}$-spaces, conditional expectation. 
TheOREM. Let $(\Omega, \mathcal{Q}, P)$ be a probability space and $1<p<\infty$. Let $T$ : $L_{p}(\Omega, \mathcal{Q}, P) \rightarrow L_{p}(\Omega, \mathcal{Q}, P)$ be a homogeneous, quasi-additive, constant preserv-

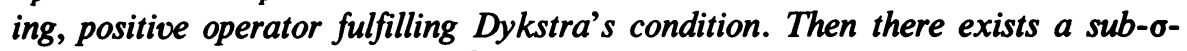
field $\mathscr{B} \subset \mathbb{Q}$ such that $T=P_{p}^{\mathscr{B}}$, i.e., $T f$ is the nearest point projection of $f$ onto the subspace $L_{p}(\Omega, \mathcal{Q}, P)$.

Proof. Let $F=\left\{f \in L_{p}(\Omega, \mathcal{Q}, P): T f=f\right\}$. Since $T$ is homogeneous and quasi-additive, $F$ is a linear space. The same properties imply that $T$ is idempotent and hence $F=\left\{T f: f \in L_{p}(\mathbb{Q})\right\}$. Now we show that $T$ is a projection onto $F$, i.e. we show

$$
\|f-T f\|_{p}<\|f-T g\|_{p} \quad \text { for } f, g \in L_{p}(\mathbb{Q}) .
$$

Using that $T$ is homogeneous, quasi-additive and that $\|I-T\|_{p}<1$ we obtain

$$
\begin{aligned}
\|f-T f\|_{p} & =\|(f-T g)-(T f-T g)\|_{p}=\|(f-T g)-(T f+T(-g))\|_{p} \\
& =\|(f-T g)-T(f+T(-g))\|_{p}=\|(f-T g)-T(f-T g)\|_{p} \\
& <\|f-T g\|_{p} .
\end{aligned}
$$

Now it remains to show that $F=L_{p}(\mathscr{B})$ for some $\sigma$-field $\mathscr{B} \subset \mathbb{Q}$. According to Proposition I-1-1 [8, p.2], this amounts to verifying that:

(i) $1 \in F$,

(ii) $F$ is closed,

(iii) $f \in F$ implies $f^{+} \in F$.

Since $T$ is constant-preserving, (i) holds. To prove (ii), let $f_{n} \in F, n \in \mathbf{N}$, and $\left\|f_{n}-f\right\|_{p} \underset{n \rightarrow \infty}{\rightarrow} 0$. Then $T f_{n}=f_{n}$ and the properties of $T$ imply

$$
\|f-T f\|_{p}=\left\|f-T f_{n}-T\left(f-T f_{n}\right)\right\|_{p} \leqslant\left\|f-f_{n}\right\|_{p} \underset{n \rightarrow \infty}{\rightarrow} 0
$$

and hence $T f=f$, i.e., $f \in F$.

To see (iii), let $f \in F$, i.e. $f=T f$. We have to prove: $T f^{+}=f^{+}$. We show at first that $T f^{+} \geqslant f^{+}$. Since $T$ is positive it suffices to show $T f^{+}>f$. As $f^{+}-f>0, T f=f$, and $T$ is positive, homogeneous and quasi-additive we obtain

$$
0<T\left(f^{+}-f\right)=T\left(f^{+}-T f\right)=T f^{+}-T f=T f^{+}-f,
$$

i.e., $T f^{+}>f$. Let $g=T f^{+}-f^{+}$. Then $g>0$ and $T g=0$. If $g>0$ on a set of positive $P$-measure, then $h=P_{p}^{\{\varnothing, \Omega\}} g>0$. Since the $p$-projection on $L_{p}(\{\varnothing, \Omega\})$ is unique we obtain $\|g-h\|_{p}<\|g\|_{p}$. Since $h \in F$ this contradicts $T g=0$. Consequently $g=0$. Hence $T f^{+}=f^{+}$, i.e., $f^{+} \in F$.

Since Bahadur's conditions directly imply our conditions, our result contains the result of Bahadur [3], who proved that a linear idempotent, selfadjoint, positive and constant preserving operator $T: L_{2}(\mathbb{Q}) \rightarrow L_{2}(\mathbb{Q})$ is a usual conditional expectation operator. It is easy to see that none of the five properties of $T$ can be dispensed without compensation.

The following example shows, that it is not possible to weaken quasi-additivity to quasi-quasi-additivity (i.e., $T(T f+T g)=T f+T g$ ), even if we add 
three other conditions which were often used by other authors, namely monotony, norm-continuity and Šidák's conditions, i.e.,

$$
T(T f \vee T g)=T f \vee T g
$$

(see [10, p. 271, Theorem 6]).

ExAmple. Let $\Omega=\{1,2\}, Q$ be the power set of $\Omega$ and $P \mid Q$ be the probability measure defined by $P(\{1\})=P(\{2\})=\frac{1}{2}$. We consider the case $p=2$. We have

$$
L_{2}(\Omega, Q, P)=\left\{\alpha 1_{\{1\}}+\beta 1_{\{2\}}: \alpha, \beta \in \mathbf{R}\right\} .
$$

For each $\alpha, \beta \in \mathbf{R}$ let

$$
c(\alpha, \beta)=\operatorname{sign}(\alpha+\beta) \frac{1}{2 \sqrt{2}} \frac{(\alpha+\beta)^{2}}{\sqrt{\alpha^{2}+\beta^{2}}} .
$$

Now define $T: L_{2}(\Omega, \mathcal{Q}, P) \rightarrow L_{2}(\Omega, \mathcal{Q}, P)$ by $T\left(\alpha 1_{\{1\}}+\beta 1_{\{2\}}\right) \equiv c(\alpha, \beta)$. Since $c(\alpha, \alpha)=\alpha, F=\{f: T f=f\}$ is the set of all constant functions, i.e., $F=L_{2}(\{\varnothing, \Omega\})$. It is easy to see that $T$ is a homogeneous, constant preserving, positive operator fulfilling Dykstra's condition. The last property immediately follows from $c(\alpha, \beta)<\alpha+\beta$ which is equivalent to $P\left((f-T f)^{2}\right)$ $<P\left(f^{2}\right)$ for $f=\alpha 1_{\{1\}}+\beta 1_{\{2\}}$ with $(\alpha+\beta)>0$.

Moreover, $T$ is idempotent, monotone, quasi-quasi-additive, fulfills Šidák's condition and is continuous. But all these properties cannot replace quasiadditivity in the preceding theorem, since $T$ is not the projection onto $F$. The projection onto $F$ is the usual conditional expectation given $\mathscr{B}=\{\varnothing, \Omega\}$, i.e.,

$$
P_{2}^{\mathscr{B}}\left(\alpha 1_{\{1\}}+\beta 1_{\{2\}}\right)=\frac{1}{2}(\alpha+\beta) .
$$

\section{REFERENCES}

[1] T. Ando, Contractive projections in $L_{p}$-spaces, Pacific J. Math. 17 (1966), 391-405.

[2] T. Ando and I. Amemiya, Almost everywhere convergence of prediction sequence in $L_{p}$ $(1<p<\infty)$, Z. Wahrscheinlichkeitstheorie und Verw. Gebiete 4 (1965), 113-120.

[3] R. R. Bahadur, Measurable subspaces and subalgebras, Proc. Amer. Math. Soc. 6 (1955), 565-570.

[4] R. G. Douglas, Contractive projections on an $L_{1}$-space, Pacific J. Math. 15 (1965), 443-462.

[5] R. L. Dykstra, A characterization of a conditional expectation with respect to a $\sigma$-lattice, Ann. Math. Statist. 41 (1970), 698-701.

[6] D. Landers and L. Rogge, On linearity of s-predictors, Ann. Probability (to appear).

[7] S. C. Moy, Characterization of conditional expectation as a transformation on function spaces, Pacific J. Math. 4 (1954), 47-63.

[8] J. Neveu, Discrete-parameter martingales, North-Holland, Amsterdam, 1975.

[9] J. Pfanzagl, Characterizations of conditional expectations, Ann. Math. Statist. 38 (1967), $415-421$.

[10] Z. Sidák, On relations between strict-sense and wide-sense conditional expectations, Theor. Probability Appl. 2 (1957), 267-271.

Fachbereich Statistik, Universität Konstanz, D-775 Konstanz, West Germany 